\title{
La vie de la SFED
}

\section{SFED's life}

\author{
G. Lesur \\ C Springer-Verlag France 2013
}

2013 n'est pas que l'année Guiseppe Verdi et Richard Wagner, tous deux nés en 1813, mais aussi celle d'un changement, non seulement à Acta Endoscopica, mais surtout au Conseil d'administration de la SFED. Après deux ans de fonction, Christophe Cellier quittera ses habits de président pour revêtir ceux de « past-président», puis plus tard d'ancien président. Je peux vous assurer que la charge de travail de président de la SFED est considérable. Qu'il soit ici remercié officiellement de son action à la tête de la société marquée par une détermination sans faille à toujours faire mieux connaître et reconnaître l'endoscopie digestive. Les soirées européennes de la SFED au succès grandissant, l'ouverture du direct de Vidéo-Digest à des collègues femmes, les actions médiatiques autour du dépistage du cancer colorectal, les relations privilégiées avec les décideurs de l'INCA, et bien d'autres actions qui vont perdurer au-delà de son mandat, c'est lui. Le temps passe mais la continuité est assurée par les hommes et la structure. Au moment où vous lirez ces lignes, un nouveau président de la SFED aura été élu par le nouveau conseil d'administration. Avec ce nouveau conseil d'administration, qui verra huit membres prendre ou pour certains reprendre leurs fonctions, la SFED va bien entendu continuer son action en faveur de l'endoscopie digestive. 2013 sera aussi l'année au cours de laquelle la SFED confiera Vidéo-Digest et le Cours Intensif, organisés par la SNFGE, à un nouvel organisateur : Europa. Soyez assurés que tout est fait dès maintenant pour que cette édition 2013, que nous souhaitons toujours plus en accord avec vos attentes, soit un succès.
Notez dès maintenant, si vous ne l'avez pas encore fait, que cette année Vidéo-Digest aura lieu en dehors des congés scolaires, du 14 au 16 novembre 2013.

La SFED va également continuer d'élaborer des recommandations telles que celles qui se trouvent dans ce numéro et qui, sous la houlette de Laurent Peyrin-Biroulet, expert reconnu, sont consacrées aux MICI. Vous trouverez également dans ce numéro les textes du symposium « L'œsophage de $\mathrm{A}$ à $\mathrm{Z}$ » organisé lors des JFHOD. L'intérêt des sujets traités et l'expertise des auteurs feront de toute évidence de ces textes des outils de référence pour beaucoup d'entre nous... et pendant au moins quelques années.

Vous l'avez sans doute remarqué, la cotisation annuelle à la SFED est passée cette année de 195 euros à 200 euros. Il ne s'agit pas d'une préférence pour les chiffres ronds, mais même si la situation financière de la SFED est satisfaisante, il faut tenir compte de l'augmentation du coût de la vie pour une cotisation qui n'avait pas été majorée depuis deux ans. Vous le savez, le paiement de cette cotisation vous donne, entre autres, la possibilité de recevoir gratuitement Acta Endoscopica et Endoscopy, et également d'adhérer à un tarif préférentiel à l'European Society of Gastrointestinal Endoscopy (ESGE), adhésion qui offre elle-même des réductions aux réunions scientifiques coordonnées par l'ESGE et dont vous trouverez la liste dans les pages « agenda » de ce numéro.

Tout le conseil d'administration de la SFED se joint à moi pour vous souhaiter de belles et fructueuses « Journées de Gastroentérologie et d'Oncologie Digestive ». Nous espérons également vous retrouver très nombreux lors du Vidéo-Digest, nouvelle cuvée 2013.

G. Lesur $(\square)$

Rédacteur en chef

hôpital Ambroise Paré, F-92100 Boulogne

e-mail : gilles.lesur@apr.aphp.fr 